FORMATION Formation emploi

Revue française de sciences sociales

103 | juillet-septembre 2008

Fuite ou mobilité des cerveaux?

\title{
L'autre réalité du programme Erasmus : affinité sélective entre établissements et reproduction sociale des étudiants
}

The other reality of the Erasmus programme: selective affinity between establishments and social reproduction of students

Die Kehrseite des ERASMUS-Programms: Affinität zwischen

Hochschuleinrichtungen und soziale Reproduktion der Studierenden

La otra realidad del programa Erasmus : afinidad selectiva entre

establecimientos y reproducción social de los estudiantes

\section{Magali Ballatore et Thierry Blöss}

\section{OpenEdition}

Journals

Édition électronique

URL : http://journals.openedition.org/formationemploi/2478

DOI : 10.4000/formationemploi.2478

ISSN : 2107-0946

Éditeur

La Documentation française

Édition imprimée

Date de publication : 1 septembre 2008

Pagination : $57-74$

ISSN : 0759-6340

Référence électronique

Magali Ballatore et Thierry Blöss, «L'autre réalité du programme Erasmus : affinité sélective entre établissements et reproduction sociale des étudiants », Formation emploi [En ligne], 103 | juilletseptembre 2008, mis en ligne le 01 septembre 2010, consulté le 30 octobre 2020. URL : http:// journals.openedition.org/formationemploi/2478; DOI : https://doi.org/10.4000/formationemploi.2478 


\section{DOSSIER}

\section{L'autre réalité du programme Erasmus : affinité sélective entre établissements et reproduction sociale des étudiants}

Magali Ballatore et Thierry Blöss*

Le programme Erasmus n'a semble-til pas atteint son objectif de réciprocité des échanges internationaux. Les étudiants mobiles constituent un ensemble fractionné de jeunes européens inscrits dans des circuits institutionnels différenciés

qui reproduisent l'ordre des inégalités de prestige entre établissements.

Le spectre des migrations étudiantes s'est élargi au cours de ces dernières années en Europe. Spontanée ${ }^{1}$ ou institutionnalisée, la mobilité géographique des étudiants désigne des durées et des issues variables, pouvant déboucher sur une installation dans le pays

\footnotetext{
${ }^{1}$ On désignera par mobilité « spontanée » la mobilité des étudiants qui ont choisi « individuellement» de s'inscrire dans un établissement d'enseignement supérieur qui ne se situe pas dans leur pays d'origine, sans convention intergouvernementale ni support institutionnel financier. La mobilité étudiante " organisée » peut être définie, quant à elle, comme une mobilité en cours d'études, incluant celle supportée par le programme communautaire Socrates II (anciennement Erasmus et Lingua II). Les échanges universitaires internationaux s'opèrent entre des organisations contractualisées en relation étroite (de recherche), et incluent en théorie une certaine réciprocité, parfois différée. Comme il est mentionné dans les documents officiels, l'action Erasmus s'adresse aux établissements d'enseignement supérieur - et donc aux étudiants et enseignants - des vingt-cinq États membres de l'Union européenne, des trois pays de l'Espace économique européen (Islande, Liechtenstein et Norvège) et des trois pays candidats (Bulgarie, Roumanie et Turquie).
}

d'accueil. Dans ce paysage de plus en plus hétérogène, où les motivations des protagonistes semblent avant tout résulter des ambitions, goûts ou choix libres et personnels des candidats au voyage, un certain nombre d'éléments d'enquête nous montrent que la question de la migration des étudiants et des diplômés résulte de logiques sociales et politiques.

* Magali Ballatore est ATER lattaché temporaire d'enseignement et de recherche) de sociologie à l'université de Provence et chercheur au LEST (Laboratoire d'économie et de sociologie du travail, UMR 6123).

Thierry Blöss est professeur de sociologie à l'université de Provence (Aix-Marseille 1) et chercheur au LEST. 
Autrement dit, les qualités individuelles ${ }^{2}$ que l'on prête aux étudiants migrants sont fortement liées à leurs caractéristiques scolaires et sociales.

${ }^{2}$ La figure dominante de l'étudiant migrant, à l'esprit bohême et festif, en quête de son identité juvénile, a été largement diffusée dans le film de Cédric Klapisch : "L'auberge espagnole ».
Cet article a pour objet le dispositif Erasmus, programme d'échanges entre étudiants au sein de la communauté européenne ( $c f$. encadré 1). Entre

Cette facette va de pair avec une appartenance sociale favorisée et la conduite de projets professionnels pour lesquels l'apprentissage d'une langue étrangère revêt une importance stratégique.

\section{Encadré 1 \\ Qui peut participer au programme Erasmus?}

Peuvent participer au programme Erasmus :

- Les établissements d'enseignement supérieur reconnus éligibles /délivrant un diplôme national de l'enseignement supérieur, un diplôme d'état ou un titre) et les établissements privés agréés par l'État. Un séjour d'études Erasmus s'inscrit obligatoirement dans le cadre d'échanges entre établissements. Aujourd'hui, les pays concernés par le programme Erasmus sont les trente pays de l'Espace économique européen (EEE) (soit les vingt sept pays membres de I'Union européenne ainsi que l'Islande, le Liechtenstein et la Norvège) et les pays candidats, soit la Turquie, la Bulgarie et la Roumanie. Chaque université fait acte de candidature en présentant les accords bilatéraux (contrats bilatéraux départementaux) signés par les coordinateurs (responsables Erasmus) et les présidents de deux universités pour un an renouvelable. Les contrats bilatéraux reposent sur des réseaux de connaissances préalablement constitués par les enseignants-chercheurs des universités.

- Les étudiants qui ont achevé leur première année d'études universitaires et qui sont citoyens de l'un des pays éligibles ou qui ont le statut de résident permanent, d'apatride ou de réfugié politique. En ce qui concerne la sélection des étudiants au sein des départements, les procédures sont très variables d'un pays à l'autre et même parfois entre les institutions d'enseignement supérieur d'un même pays, voire d'une même université. Ce qui est commun à l'ensemble des départements des universités françaises, c'est le rôle décisif et central que joue le responsable pédagogique dans l'attribution des places. Le choix des candidats est laissé en dernière instance à l'appréciation du responsable Erasmus, unique dans chaque département. De manière générale, nous pouvons dire que ce système de "recrutement » des étudiants Erasmus diffère du système anglais hautement structuré par avance et transparent ou du système bureaucratique et agencé italien. En Italie, les postes disponibles et les destinations pour le déroulement d'un séjour d'études à l'étranger sont publiés chaque année par avis de concours. Les commissions de facultés évaluent les demandes des candidats, rédigent les listes par ordre de mérite pour chaque destination, attribuant à chaque étudiant un nombre de points. Ces listes sont envoyées au bureau Erasmus, qui réattribue automatiquement les places des étudiants qui ont renoncé. En Angleterre, le programme Erasmus a permis à des départements, dans un système de plus en plus concurrentiel et pragmatique, de créer de nouveaux programmes combinés "d'études en Europe Continentale » (Bs or Ms with Study in Continental Europel pour attirer et/ou absorber une partie de l'augmentation du flux d'étudiants, tout en maintenant le mythe de l'égale valeur de toutes les formations. Chaque département de chaque université possède ses propres exigences en termes de résultats obtenus aux A'levels pour l'admission et ces exigences peuvent différer d'un programme à l'autre.

- Les enseignants de l'enseignement supérieur et, uniquement dans le cadre des réseaux thématiques (volet 3 d'Erasmus), les organismes privés et publics qui coopèrent avec les établissements d'enseignement supérieur (centres et organismes de recherche, associations, collectivités locales, entreprises, partenaires sociaux, OGN - Organisation non gouvernementale). 


\section{Encadré 2}

\section{Le programme Erasmus : quelques dates et événements clés}

Le programme Erasmus est né d'une décision du Conseil européen du 15 juin 1987 (87/327/EEC). La première phase durera trois ans. La seconde phase, établie par une décision du Conseil du 14 décembre 1989, couvre cinq années académiques, de 1990/91 à 1994/95. II intégrera, à partir de 1995, le principal programme pour le développement d'une dimension européenne de l'enseignement de la Commission européenne : Socrates (dont l'enveloppe financière pour la période 1995-1999 s'élève à 850 millions d'euros dont au moins 55 \% seront strictement consacrés au programme Erasmus).

Erasmus représente le volet consacré à l'enseignement supérieur du programme Socrates II ${ }^{*}$ ). Ce dernier soutient les projets de coopération européenne dans huit domaines, de l'éducation scolaire à l'enseignement supérieur, en passant par les nouvelles technologies et la formation permanente. Le programme baptisé Socrates, regroupe les actions suivantes: Comenius (pour l'enseignement primaire et secondaire), Erasmus (enseignement universitaire), Grundtvig (pour la formation d'adultes principalement), Lingua lqui concerne l'apprentissage et l'enseignement des langues de l'Europe), Minerva (pour ce qui est des technologies de l'information et de la communication en éducation) et tous les programmes qui concernent l'observation et l'innovation des systèmes d'éducation et des politiques, les actions jointes avec d'autres programmes européens et les mesures d'accompagnement.

Le système ECTS (European credit transfert system), pour garantir la reconnaissance mutuelle des diplômes en Europe, a été lancé en 1988, après un appel à candidature aux universités publiques Uournal officiel des Communautés européennes C 197/11 du 27 juillet 1988).

Plus récemment, le 17 juillet 2002, le Commission européenne a proposé Erasmus World, dont l'objectif est de contribuer à un enseignement de "qualité » au sein de l'Union européenne, qui favorise la coopération avec les pays tiers. Doté d'un budget de 200 millions d'euros, il s'étend de 2004 à 2008, avec comme principaux objectifs la création de master, le développement de bourses d'études, l'augmentation de l'attractivité de l'enseignement supérieur européen pour les pays tiers.

Les deux principales déclarations mentionnant les objectifs de la Communauté européenne en matière d'enseignement supérieur et de mobilité sont :

- La déclaration de Paris, le 25 mai 1998. Les ministres de l'éducation français, italien, britannique et allemand ont signé une première déclaration présentant les bases de l'harmonisation des systèmes d'enseignement sur lesquelles repose la déclaration de la Sorbonne. Ils ont souligné le désir de " respect de la diversité », mais également l'engagement vers un cadre formatif commun, pour notamment améliorer la reconnaissance externe du système d'enseignement européen et favoriser la mobilité des étudiants.

- La déclaration de la Sorbonne, le 19 juin 1999, réaffirme les objectifs de la construction d'une Europe de la connaissance.

Le Journal officiel des Communautés européennes (C371/4) du 23 décembre 2000 comportait une résolution du Conseil et des représentants des gouvernements des États membres qui résume les priorités concernant la mobilité en Europe : "... Accueillent favorablement le plan d'action pour la mobilité repris en annexe et présenté aux ministres de l'Éducation à la Sorbonne, le 30 septembre 2000. Ce plan répond à trois grands objectifs: Définir et démocratiser la mobilité en Europe; Promouvoir les formes de financement adéquates; Accroître la mobilité et en améliorer les conditions. »

(*) : Peu après le lancement du programme Erasmus, un groupe de pays scandinaves créa le NORDPLUS programme pour la mobilité étudiante au sein de cette aire géographique. D'autres programmes virent aussi le jour ces deux dernières décennies, comme le STIR (programme incitatif pour l'internationalisation) le PSO (le programme pour la coopération avec les pays de l'Europe de l'Est), le GROS (un programme de coopération régionale). 
1987, année de naissance du Programme, et 2004, plus d'un million d'étudiants européens ont effectué un séjour à l'étranger dans le cadre d'Erasmus. Rapportée à la population étudiante de l'Europe, la population Erasmus reste néanmoins minoritaire: seuls $1 \%$ des étudiants européens ont bénéficié de ce dispositif. Le caractère sélectif du programme constitue donc le cadre général de notre propos qui s'appuie sur une analyse comparative entre trois pays: la France, la Grande-Bretagne et l'Italie. Ainsi, en dépit d'un principe de réciprocité des échanges, les relations entre les institutions universitaires se fondent sur des logiques d'«affinités sélectives » qui à la fois révèlent l'élitisme relatif du dispositif et renforcent les déséquilibres migratoires existants entre les pays concernés. Plus généralement, cet article vise à démontrer que la mobilité géographique des étudiants est liée à la diversification croissante des parcours étudiants mais aussi à la sélection sociale accrue des systèmes d'enseignement supérieur : les usages que font les étudiants de ce programme, les choix de destination à l'étranger, leurs projets scolaires ou encore leur propre expérience biographique constituent, de ce point de vue, autant de facteurs qui mettent en discussion le principe démocratique du dispositif officiellement reconnu ou attendu ${ }^{3}$.

\section{ERASMUS REPRODUIT LES DÉSÉQUILIBRES DES FLUX MIGRATOIRES}

La création du programme Erasmus et plus largement la mise en place de protocoles d'échange d'étudiants au sein de l'Union européenne ont incon-

\footnotetext{
${ }^{3}$ Entre1987 et 2004, plus d'un million d'étudiants ont effectué un séjour à l'étranger dans le cadre d'Erasmus, suscitant ainsi l'enthousiasme des membres de la Commission européenne. Son président, Romano Prodi, se félicitait, dans un communiqué, du succès du dispositif dans ces termes : «Aujourd'hui, les étudiants Erasmus sont libres de choisir parmi l'un des 30 pays participants. Ils contribuent à forger une identité européenne commune. Leurs histoires nous montrent qu'après quinze ans, Erasmus reste une réussite capitale de l'Union européenne. " D'autres déclarations officielles insisteront sur la démocratisation en cours des possibilités de séjours à l'étranger ( 750 millions d'euros étant réservés aux bourses accordées aux étudiants pour la période 2000-2006) et sur la résorption du déséquilibre séculaire des migrations.
}

testablement révélé que si la mobilité étudiante n'est pas un phénomène récent, l'essentiel de ses flux était jusque-là essentiellement tourné ailleurs que vers l'Europe et concernait pour majorité des étudiants non européens. Selon les données de l'OCDE (Organisation de coopération et de développement économiques), la mobilité étudiante internationale se concentre dans cinq nations: les USA (28\% d'étudiants étrangers), le Royaume-Uni (12\%), l'Allemagne (11\%), la France (10\%) et l'Australie (9\%) attirent 8 étudiants étrangers sur 10 . Selon ces mêmes sources, il y aurait 1,5 million d'étudiants étrangers dans les pays de l'OCDE, dont $56 \%$ proviennent de pays extérieurs à la zone OCDE. Le groupe national le plus important est représenté par les étudiants chinois, suivis des coréens et des japonais. En 2003-2004, 245300 étudiants étrangers poursuivent des études dans l'enseignement supérieur français, et 20275 entrent par le programme Erasmus. Le programme Erasmus ne représente que $8,2 \%$ de l'ensemble des mobilités entrantes cette année-là en France.

L'attractivité des universités européennes existe pourtant, mais elle est sélective. Celles qui accueillent le plus grand nombre d'étudiants étrangers, y compris non européens, sont dans l'ordre allemandes, françaises et anglaises. Selon l'OCDE, en 2003, $10 \%$ des étudiants inscrits dans un établissement d'enseignement supérieur français étaient étrangers. Alors qu'entre les années académiques 1990-91 et 1998-99 le nombre d'étudiants étrangers avait chuté de 136300 à 122 100, depuis, leur effectif progresse à un rythme annuel d'environ $12 \%$, supérieur à celui des étudiants français (Fabre et Tomasini, 2006). À l'université stricto sensu, les étudiants étrangers représentent $13,7 \%$ des effectifs en 2003. Particulièrement nombreux en troisième cycle (où un étudiant sur quatre est étranger), ils sont également plus nombreux dans des filières comme «Économie et AES » (Administration économique et sociale), «Lettres et Sciences humaines» et «Sciences ». En Angleterre les étudiants étrangers représentaient $12 \%$ de l'ensemble des inscrits en 2003, à un niveau donc comparable à la France ${ }^{4}$. Le

\footnotetext{
${ }^{4}$ Dinstinguons cependant deux segments de l'enseignement supérieur britannique : universitaire « higher education 》 et postscolaire «further education ». Plus nombreux dans le premier secteur,.../...
} 
cas italien se démarque nettement, puisque seuls $2 \%$ d'étudiants étrangers sont inscrits à l'université cette même année, concentrés essentiellement en Italie septentrionale, et que paradoxalement la proportion d'inscrits au niveau du troisième cycle universitaire est plutôt faible, contrairement à ses deux « voisins » européens.

Même si la mobilité étudiante en Europe est difficile à appréhender ${ }^{5}$, elle semble en constante augmentation, à l'instar du programme Erasmus (Kelo et al., 2006 ; OCDE, 2007). Cependant, toutes les nations

.../... les étudiants étrangers représentent $11 \%$ des inscrits à plein temps du premier niveau universitaire «full-time first degree », mais $65 \%$ des étudiants à plein temps au niveau master " full-time taugh postgraduate » et $48 \%$ des étudiants en début de carrière académique "full-time research degree students ».

${ }^{5}$ En France, il n'existe pas de système national de recueil systématique de données statistiques concernant les étudiants à l'étranger, ni de dispositif statistique permettant de recenser et de suivre l'évolution de la mobilité internationale des étudiants et des personnels de recherche français. D'autres pays en font une estimation à partir de plusieurs sources. En Italie, on citera par exemple l'AIRE (Anagrafe Italiani Residenti all'Estero), au Royaume-Uni les Councils pour le financement de l'enseignement supérieur (HEFCE, Higher Education Funding Council for England, HEFCW, Higher Education Funding Council for Wales par exemple), ou encore le Council for international Education. L'immatriculation auprès des services consulaires n'étant dans aucun de ces pays obligatoire, elle ne permet pas de fournir des estimations fiables pour ces populations. Seules les données issues des déclarations des pays membres de l'OCDE et des programmes de mobilité européens permettent d'obtenir une estimation, avec toutes les difficultés que cette mesure comporte, car chaque pays et chaque centre statistique adopte (comme pour le chômage) ses propres critères pour définir et recenser les étudiants mobiles. européennes ne connaissant pas le même niveau de mobilité ni ne suivent les mêmes orientations. Les étudiants britanniques par exemple sont assez peu mobiles et apparaissent comme peu tournés vers l'Europe (cf. Sussex Centre for Migration Research et Center for Applied Population Research, 2004) les États-Unis étant leur destination privilégiée. En sens inverse, la Grande-Bretagne apparaît comme le premier pays destinataire des étudiants de l'Union européenne, tout particulièrement des jeunes français (cf. tableau 1).

La France se caractérise par un déséquilibre relativement réduit entre étudiants sortants et étudiants entrants. L'Italie pour sa part accueille peu d'étudiants en mobilité internationale, comparativement à la France et surtout à la Grande-Bretagne. Et les migrations internationales des italiens semblent davantage concerner les diplômés après leurs études que les étudiants. Une étude relativement récente (Becher et al., 2002) signale un accroissement important du nombre de diplômés italiens travaillant à l'étranger (représentant environ 2,3\% de l'ensemble des diplômés nationaux, alors que les taux des pays méditerranéens comme la France et l'Espagne sont respectivement de $0,9 \%$ et de $0,7 \%$, sur la même période).

La mobilité institutionnalisée dans le cadre du programme Erasmus reproduit les préférences séculaires entre pays. À ce titre, les principales destinations des étudiants Erasmus français sont la Grande-

Tableau 1

Entrée des étudiants en Grande-Bretagne selon leur pays d'origine en 2003-2004

\begin{tabular}{|l|r|r|}
\hline \multicolumn{1}{|c|}{ Pays d'origine } & Effectifs & En \% \\
\hline France & 4652 & 28 \\
Allemagne & 3159 & 19 \\
Espagne & 2974 & 18 \\
Italie & 1511 & 9 \\
Hollande & 635 & 4 \\
Finlande & 552 & 3 \\
Suède & 494 & 3 \\
Belgique & 341 & 2 \\
Danemark & 330 & 2 \\
\hline \multicolumn{2}{|c|}{ TOTAL (pour l'ensemble des pays participants) } & 16627 \\
\hline
\end{tabular}

Source : http://ec.europa.eu/education/programmes/socrates/erasmus/stat_fr.html 
Bretagne, l'Espagne et l'Allemagne. Les britanniques se rendent majoritairement en France, en Espagne et en Allemagne. Quant aux étudiants Erasmus de nationalité italienne, ils privilégient l'Espagne, la France et l'Allemagne. Une des études statistiques consacrées à la mobilité étudiante à l'intérieur des pays de l'Union européenne (Jallade et $a l ., 1996)^{6}$ soulignait déjà le déséquilibre existant en montrant combien des États membres comme la Grande-Bretagne, l'Irlande et dans une moindre mesure l'Espagne accueillaient plus d'étudiants qu'ils n'en envoyaient, l'Italie à l'opposé, dont la langue a un statut international moins important, se présentant comme un pays essentiellement « exportateur ». Plus récemment, les statistiques officielles sur les étudiants sortants confirment la représentation dominante de pays comme l'Allemagne, la France et l'Espagne dans la population Erasmus, les étudiants britanniques n'étant que faiblement présents (cf. tableau 2 : «Étudiants sortants »). Quant aux destinations en jeu, elles confirment le rôle moteur de l'Espagne et de la France dans la vitalité des échanges Erasmus ( $c f$. tableau 2 : «Étudiants entrants »). La réalité des « déséquilibres » migratoires qui en résulte illustre, de ce point de vue, combien la réciprocité des échanges reste encore un objectif à atteindre.

${ }^{6}$ Les dispositifs étudiés concernent les programmes Erasmus et Lingua II en 1994-1995.
En essor constant en France, la mobilité étudiante intra-européenne mise en place par le programme Erasmus représente près des trois-quarts de l'ensemble de la mobilité organisée dans l'hexagone. Elle s'ajoute aux programmes d'échanges avec les pays non européens ${ }^{7}$. Cette mobilité peut être décrite comme une mobilité «à court terme ». Comparée à la mobilité spontanée qui englobe les étudiants ayant choisi de suivre un cursus entier à l'étranger, la mobilité organisée se déroule sur une période ne pouvant excéder neuf mois. Elle s'inscrit dans le curriculum du pays d'origine, de telle sorte que l'étudiant obtient, à l'issue de son séjour à l'étranger, le diplôme de son université d'origine et non celui de son université d'accueil. Cela constitue l'originalité principale de ce type de mobilité institutionnalisée?.

\footnotetext{
${ }^{7} C f$. le programme Crepuq (Conférence des Recteurs et des Principaux des Universités du Québec) par exemple, deuxième programme le plus important (en nombre d'étudiants qui y participent en France), après Socrates. Il est à noter qu'à la différence du programme d'échanges Erasmus, la sélection des individus se fait par l'université d'accueil à partir de dossiers constitués individuellement et envoyés par l'étudiant directement à l'université de son choix.

${ }^{8}$ L'autre caractéristique de la mobilité instituée par le programme Erasmus est sa relative facilité de mesure, puisque par le biais des agences Socrates nationales et des services des relations internationales des universités, l'ensemble des «entrants" et des « sortants » est, depuis la création du programme en 1987, enregistré systématiquement chaque année.
}

Tableau 2

La mobilité étudiante - Programme Erasmus, 2004-2005

\begin{tabular}{|l|c|c|c|c|c|}
\cline { 2 - 6 } \multicolumn{1}{c|}{} & \multicolumn{2}{c|}{ Étudiants sortants } & \multicolumn{2}{c|}{ Étudiants entrants } & $\begin{array}{c}\text { Ratio entrants/ } \\
\text { sortants }\end{array}$ \\
\hline & Effectifs & $\begin{array}{c}\text { \% de l'ensemble } \\
\text { des 24 pays }\end{array}$ & Effectifs & $\begin{array}{c}\text { \% de l'ensemble } \\
\text { des 24 pays }\end{array}$ & (Base 100) \\
\hline France & 21561 & $\mathbf{1 5}$ & 20519 & $\mathbf{1 4}$ & $\mathbf{9 5}$ \\
\hline Italie & 16440 & 11 & 13370 & 9 & 81 \\
\hline Grande-Bretagne & 7214 & 5 & 16266 & 11 & 225 \\
\hline $\begin{array}{l}\text { Allemagne } \\
\text { Espagne } \\
\text { Finlande }\end{array}$ & 22427 & 16 & 17273 & 12 & 77 \\
$\ldots$ & 20819 & 14 & 25511 & 18 & 123 \\
\hline Ensemble des pays & 144037 & 3 & 5351 & 4 & 136 \\
\hline
\end{tabular}

Le tableau se lit ainsi : $15 \%$ des étudiants Erasmus européens sont de nationalité française. 14 \% des étudiants Erasmus européens ont choisi la France comme destination. Cette même année, en France, pour 100 étudiants Erasmus sortants, il y avait 95 entrants.

Source : http://ec.europa.eu/education/programmes/socrates/Erasmus/stat_fr.html 


\section{ENTRE POLITIQUES NATIONALES DE FORMATION ET RÉSEAUX D'ÉTABLISSEMENTS}

Les échanges Erasmus se basent sur des contrats bilatéraux signés par des enseignants-chercheurs de deux départements, avec l'accord des Services de relations internationales des universités concernées. Ils recouvrent ainsi les hiérarchies et connivences existantes entre les institutions et les pays. La «fermeture progressive» des universités britanniques rend ce phénomène plus visible. Ainsi tous les étudiants européens ne se trouvent pas placés devant la même offre de mobilité. En Italie, les inégalités territoriales et en France le système dual ont un impact sur la quantité et la "qualité » des contrats qui sont noués entre les départements des universités.

\section{Diminution sélective des échanges pratiqués outre-manche}

Depuis la restriction du nombre de contrats en Grande-Bretagne, à la fin années 90, réclamée par les autorités universitaires (pour des raisons budgétaires, étant donné le déséquilibre constaté entre entrants et

\section{Encadré 3 \\ Déroulement et calendrier de l'enquête de terrain}

Cet article est issu d'une enquête comparative par étude de cas entre trois pays (Angleterre, France, Italie) qui appartiennent à des aires géographiques différentes aux traditions migratoires et académiques différentes. Le choix de ces trois pays, berceaux des universités, a été déterminé par la différenciation que l'on peut opérer entre pays exportateurs et pays importateurs en matière d'échanges et de mobilité. En ce qui concerne le programme Erasmus, les déséquilibres sont mis en lumière par Maiworm et Teichler (1997) notamment. Le choix des institutions universitaires pour l'étude de cas a consisté à prédéfinir des traits communs, des critères pour éviter un certain nombre de biais dans la comparaison. Nous avons donc choisi des universités pluridisciplinaires dans des grandes villes de province d'une taille à peu près identique, en éliminant les universités les plus réputées sur le plan international. Nous avons privilégié des universités anciennes, dont la création est antérieure aux années de la massification "scolaire » et qui possèdent des centres de recherche.

L'enquête réalisée a pour caractéristique principale de croiser méthodes qualitative et quantitative :

\section{- Une enquête par questionnaires :}

Principalement, une enquête par questionnaires a été menée auprès d'étudiants " sortants » des trois universités européennes ayant participé au programme Erasmus en 2004-2005, interrogés à leur retour dans l'institution d'origine. En complément des questionnaires, une enquête quantitative de même ampleur a été menée dans ces trois universités auprès d'une population témoin (" sédentaire ", composée d'étudiants en première et deuxième années interrogés à la sortie de cours, à l'entrée des bibliothèques ou des lieux de restauration). Au total, comme le récapitule le tableau ci-dessous, 758 questionnaires ont ainsi été recueillis :

\begin{tabular}{|l|c|c|c|}
\hline \multicolumn{1}{|c|}{ Tableau 1 } & Population « Erasmus » & Population « témoin » & Total \\
\hline Université de Provence & 155 & 105 & 260 \\
\hline Université de Turin & 127 & 153 & 280 \\
\hline Université de Bristol & 82 & 136 & 218 \\
\hline Total & 364 & 394 & 758 \\
\hline
\end{tabular}




\section{Encadré 3 (suite)}

Ce qui correspond, à l'université de Provence, à un traitement de plus de la moitié de la population Erasmus sortante en 2004-2005, à un peu plus d'un tiers à l'université de Bristol et à environ 20 \% de la population Erasmus sortante de l'université de Turin. On pouvait recenser 277 étudiants sortants à l'université de Provence en 2004-2005, 243 à l'université de Bristol et 733 à l'université de Turin.

\section{- Une enquête par entretiens (2004-2005) :}

L'enquête s'appuie également sur l'exploitation de 58 entretiens semi-directifs (entre trois-quarts d'heure et une heure et demil, menés auprès des étudiants Erasmus, des responsables enseignants-chercheurs du programme, des responsables administratifs des universités.

sortants), les enseignants-chercheurs britanniques ont conservé leurs échanges avec les «universités culturellement et traditionnellement proches des standards britanniques », selon les termes du responsable Erasmus d'un département de Sciences humaines de l'université de Bristol. Dans le même temps, la plupart des responsables Erasmus, hors départements de langues, déclarent avoir réduit, voire rompu leurs contrats avec des institutions au sein desquelles leurs étudiants étaient «mal accueillis » ou «mal suivis» ou encore dont les programmes étaient trop éloignés de ceux de leur université. L'évolution des effectifs de la population étudiante Erasmus au cours de ces dernières années révèle ainsi la particularité de la situation de la Grande-Bretagne qui n'a cessé de réduire sa participation, à l'inverse des tendances observées dans les autres pays: les jeunes italiens et français étant chaque année plus nombreux à faire l'expérience du séjour à l'étranger ( $C f$. graphique 1).

\section{Graphique 1}

Mobilité étudiante sortante : programme ERASMUS 1999-2004

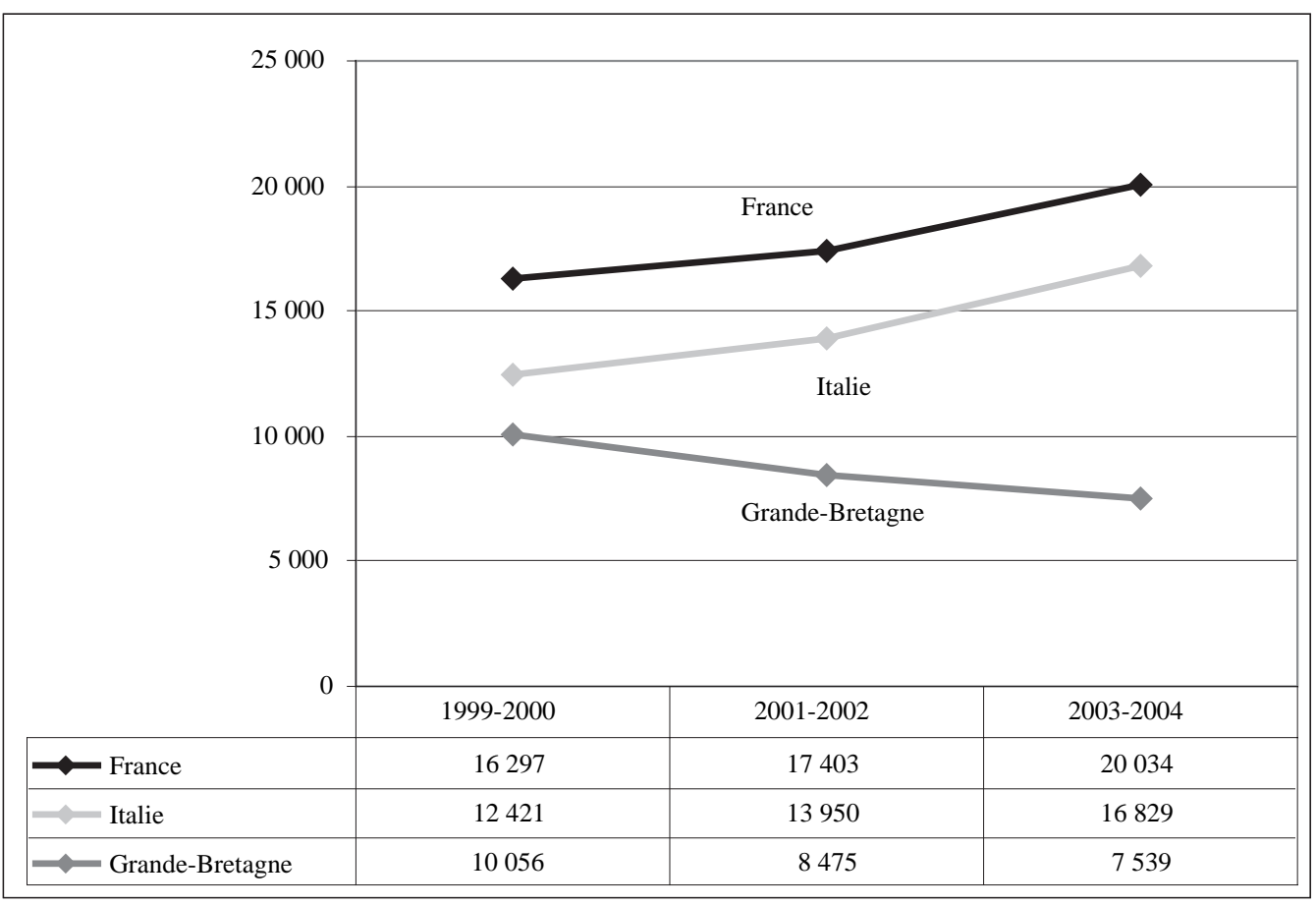

Source : http://ec.europa.eu/education/programmes/llp/erasmus/stat_en.html 
Entre 1999-2000 et 2001-2002, le nombre d'étudiants britanniques sortants est passé de 10056 à 8475 et le nombre d'étudiants entrants de 20685 à $17660^{\circ}$. Si l'on raisonne en proportion, en considérant le poids de chaque nationalité dans l'évolution de ce programme, on constatera de façon encore plus nette à quel point la part des étudiants britanniques dans la population étudiante Erasmus a sensiblement chuté : $-46,4 \%$ entre 1999 et 2005 , alors que face à la présence croissante des autres nationalités européennes, les jeunes italiens et français maintiennent quasiment leur position, autour de $12 \%$ et $15 \%$.

Ce processus de diminution sélective des échanges pratiqué outre-manche peut s'illustrer par la politique d'un département de «Sciences et structure de la matière » que nous avons visité. Les échanges de ce département se font uniquement en direction de la France, avec des écoles d'ingénieurs, seules à même, selon les dires du responsable Erasmus local, de garantir une qualité des études équivalente voire supérieure à celle du département de son université. Vers l'Italie, les échanges s'opèrent majoritairement avec des universités anciennes (Bologne, Sienne), à la renommée bien établie, les universités du Mezzogiorno ${ }^{10}$, moins cotées, peinant «à recruter » parmi les étudiants britanniques.

Même les départements de langue des universités britanniques, soucieux de maintenir leurs contrats avec le plus grand nombre d'universités européennes (le séjour d'un an à l'étranger étant obligatoire pour un étudiant qui prépare une Licence), ont vu, dans la période récente, le nombre de leurs étudiants Erasmus diminuer au profit de formes de mobilité de plus en plus appréciées: les stages professionnels (work placements) à l'étranger, d'une durée de 3 à 12 mois, qui permettent à la fois aux étudiants britanniques d'obtenir un revenu financier et aux universités de tutelle de ne pas être contraintes par des obligations d'échange ${ }^{11}$. Il existe plusieurs formes de mobilité

\footnotetext{
${ }^{9}$ Selon le rapport final du SCRE (The Scottish Council for Research in Education) publié en avril 2004.

${ }^{10}$ De l'Italie du sud.

11 Dans les départements de langues, les enseignants notent que le développement des postes d'assistants en langues (language assistantships) dans l'enseignement secondaire fait du séjour Erasmus un second choix pour beaucoup d'étudiants. Dans les universités britanniques, la baisse de la demande pourrait ainsi conduire à réduire encore davantage le nombre de places Erasmus $\quad . . / \ldots$
}

institutionnalisée non Erasmus dans l'enseignement supérieur britannique. L'association pour les échanges d'étudiants pour des expériences techniques : (IAESTE - Association for the Exchange of Students for Technical Experience) permet par exemple à des étudiants en Sciences et ingénierie de participer à des échanges durant leurs vacances scolaires. Les étudiants de langues peuvent partir et être rémunérés en tant qu'assistants de langues. Des stages courts rémunérés, inclus dans la formation initiale, sont aussi proposés par certains départements. D'après le rapport de deux centres de recherches en migration britannique $^{12}$, qui compare les tendances de la mobilité Erasmus et non Erasmus, l'augmentation de la mobilité non Erasmus (essentiellement dirigée vers l'Amérique du nord et l'Australie, hormis pour les étudiants en Langues), compense la chute de la mobilité Erasmus. Le rapport précise que ce sont les universités récentes qui ont le plus recours à la mobilité institutionnalisée rémunérée non Erasmus.

Ce processus d'affinité sélective fait que l'université de Bristol, bien classée dans la hiérarchie des universités européennes, a maintenu principalement des liens avec d'autres universités ou institutions en Europe qui lui ressemblent. A contrario, en visitant les sites internet des universités nées de la massification scolaire, on s'aperçoit qu'il en va tout autrement. C'est ainsi, à titre d'exemple, que les départements de « Sciences et Structures de la Matière » de l'université de Sussex ont des contrats avec des universités françaises créées elles aussi dans les années 60 pour faire face à l'augmentation du nombre de bacheliers.

\section{Erasmus : un révélateur des différences Nord-Sud en Italie}

L'exemple italien atteste également de cette sélectivité en réseau: l'école polytechnique de Turin («politecnico di Torino »), réputée historiquement pour avoir formé les ingénieurs de la FIAT, a de

\footnotetext{
.../... disponibles au sein des départements (pour les étudiants sortants et par conséquent pour les étudiants entrants).

12 Report by the Sussex Centre for Migration Research, University of Sussex, and the Centre for Applied Population Research, University of Dundee, International student mobility, Commissioned by HEFCE, SHEFC, HEFCW, DEL, DfES, UK Socrates Erasmus Council, HEURO, BUTEX and the British Council, July 2004/30, Issues paper, $115 \mathrm{p}$.
} 
nombreux contrats avec des écoles d'ingénieurs françaises, alors que l'université polytechnique de Bari (localisée dans la région des Pouilles, au sud de l'Italie), par exemple, n'enregistre que de rares échanges avec des écoles d'ingénieurs françaises de haut niveau. Ainsi, d'après l'étude de l'Observatoire statistique de l'université de Bologne sur 40000 diplômés de l'année 1999, conduite dans 18 universités italiennes sur tout le territoire, les établissements possédant le pourcentage le plus élevé de diplômés Erasmus (supérieur à 10 \%) se situaient tous au nord de Rome (Cammelli, 2001).

En dehors, là encore, des départements de langues, les enseignants-chercheurs responsables Erasmus se réfèrent à une communauté de culture pour justifier leurs choix. À ce titre, l'Espagne se révèle être la première destination des étudiants Erasmus italiens. «Culturellement» plus proches, ce pays serait organisé de manière similaire et permettrait aux étudiants italiens de pouvoir suivre des enseignements correspondant plus étroitement à ceux de leur université d'origine. Au-delà des affinités culturelles affichées qui justifient les choix opérés, les critères d'excellence fortement induits par la compétition économique comptent aussi. Dans ce contexte, la GrandeBretagne (et sa langue internationalement reconnue) constitue, comme déjà indiqué, une destination de choix pour les jeunes européens ( $c f$. Tableau 1).

Intériorisant les critères de qualité de la compétition économique internationale, les étudiants sont en effet de plus en plus nombreux à vouloir se rendre dans des pays anglophones et le peu de places dont disposent les départements visés oblige à une sélection plus forte des étudiants. Cela renforce les modalités de la sélection scolaire : les étudiants qui se rendent en Grande-Bretagne ont en général un parcours scolaire rapide et brillant, alors que ceux qui se dirigent vers les pays d'Europe de l'Est ou tout autre pays dont la langue nationale est minoritaire ne sont en général pas ou peu sélectionnés (pour les procédures de sélection, $C f$. Encadré 1).

\section{Les affinités électives} dans le système dual français

En France enfin, on observe aussi ce phénomène d'affinité sélective. En confrontant les deux secteurs du système d'enseignement supérieur que sont les universités et les Grandes Écoles, on retrouve aussi ces mêmes liens d'affinités qui se fondent sur des réputations héritées d'une longue histoire. L'université, qui a accueilli les étudiants de la massification scolaire, a en définitive assez peu bénéficié de l'ouverture vers l'international, en comparaison avec les Grandes Écoles. En 2003, il y avait environ 50000 étudiants français en mobilité dans la zone OCDE. Parmi eux, 9231 provenaient d'une Grande École et 12079 étaient des élèves ingénieurs effectuant un stage à l'étranger ${ }^{13}$. Plus de $40 \%$ des étudiants mobiles proviennent donc des Grandes Écoles et autres écoles d'ingénieurs, lesquelles ne totalisent qu'environ $5 \%$ des effectifs de l'enseignement supérieur français. Les étudiants mobiles sont ainsi largement surreprésentés dans ces établissements. Leur recrutement est de ce point de vue emblématique des logiques de sélection scolaire et de leur évolution, en l'occurrence de la dualité entre élèves des Grandes Écoles et étudiants des universités, dont les premiers sortent par conséquent largement gagnants. Sans compter que les destinations choisies renforcent là encore la diversification sociale des parcours étudiants et ses effets sur les futures trajectoires scolaires, migratoires et professionnelles.

\section{ÉLABORER UN PROJET PROFESSIONNEL... OUDÉVELOPPER LA CONFIANCE EN SOI?}

Dans leur très grande majorité, les étudiants Erasmus britanniques interrogés, et dans une moindre mesure les jeunes français, expriment le souhait de travailler dans leur propre pays, quel que soit le secteur d'activité professionnelle ( $c f$. Tableau 3). Seulement

\footnotetext{
${ }^{13} \mathrm{La}$ Commission internationale de la conférence des Grandes Écoles a procédé à une première enquête en 2003. Les résultats indiquent que 9231 étudiants étaient en mobilité à l'étranger, dont $22 \%$ en formation pour l'obtention d'un co-diplôme. Près de $48 \%$ de la mobilité internationale s'opère vers l'Europe de l'ouest, $26 \%$ vers l'Amérique du Nord, 8,4\% vers les pays scandinaves, 7,8\% vers le Moyen-Orient, 6,7\% vers l'Amérique Latine. Trois pays arrivent en tête des destinations : le RoyaumeUni, les États-Unis et le Canada; viennent ensuite l'Espagne et l'Allemagne.
} 
Tableau 3

Projets scolaires et professionnels des étudiants Erasmus (en \%)

\begin{tabular}{|l|c|c|c|}
\cline { 2 - 4 } \multicolumn{1}{c|}{} & \multicolumn{3}{c|}{ Étudiants ERASMUS } \\
\cline { 2 - 4 } \multicolumn{1}{c|}{} & $\begin{array}{c}\text { Université } \\
\text { de Provence }\end{array}$ & $\begin{array}{c}\text { Université } \\
\text { de Turin }\end{array}$ & $\begin{array}{c}\text { Université } \\
\text { de Bristol }\end{array}$ \\
\hline Projet professionnel précis - secteur privé - dans le pays d'origine & 38 & 32 & 61 \\
Enseignement ou concours - secteur public - dans le pays d'origine & 27 & 5 & 10 \\
Création d'entreprise & 1 & 0 & 0 \\
Poursuite des études à l'étranger & 1 & 13 & 2 \\
Stage ou travail à l'étranger & 9 & 18 & 5 \\
Recherche dans le pays d'origine ou à l'étranger & 5 & 8 & 5 \\
Pas de projet scolaire ou professionnel précis & 20 & 24 & 17 \\
\hline Total & 100 & 100 & 100 \\
\hline
\end{tabular}

Le tableau se lit ainsi : $38 \%$ des étudiants Erasmus français interrogés (issus de l'université de Provence) ont un projet professionnel dans leur propre pays, contre $32 \%$ des étudiants italiens et $61 \%$ des étudiants britanniques.

Source : enquête par questionnaires (cf. encadré 3).

7,3\% d'entre eux évoquent la possibilité de quitter la Grande-Bretagne, le plus souvent suivant un cursus de langues étrangères, pour poursuivre des études ou obtenir un emploi (et $10 \%$ en France). Ce qui est bien loin des aspirations de leurs homologues italiens qui sont plus de $30 \%$ à souhaiter prolonger l'expérience de mobilité.

Pour les étudiants Erasmus britanniques originaires de l'université de Bristol, le séjour à l'étranger représente donc moins fréquemment un moyen d'élargir leur horizon professionnel ou leur employabilité que pour les étudiants Erasmus des universités de Provence et surtout de Turin. Globalement par conséquent, les statistiques du programme Erasmus montrent un déclin non négligeable de la participation britannique. Les auteurs du rapport du Centre de recherche SCRE (Pirrié et al., 2004) l'expliquent notamment par un possible accroissement de la participation à d'autres formes de mobilité internationale comme les programmes bilatéraux ou les dispositifs d'échanges existant avec des pays extra-européens. Les faibles compétences et/ou intérêt des jeunes britanniques pour les langues étrangères pourraient également être considérés comme un facteur explicatif important (leur taux de mobilité étant un des plus bas d'Europe en rapport avec l'ensemble de la population étudiante). À Bristol, les étudiants
Erasmus inscrits en Licence (Bachelor's degree) doivent effectuer ce séjour à l'étranger en troisième année de leur cursus. Or pour valider cette Licence, l'année d'obtention du diplôme doit obligatoirement être réalisée à l'université de Bristol. Dès lors, les étudiants Erasmus sont contraints d'effectuer une année supplémentaire, c'est-à-dire d'obtenir leur Licence en quatre ans. Le séjour à l'étranger a ainsi pour eux très peu de poids, voire aucun, dans la validation des études (il compte pour moins de $20 \%$ de la note finale), en dépit de l'existence officielle d'un système de reconnaissance mutuelle des diplômes européens : «ECTS (European credit transfert system)». Les Erasmus coordinators énoncent souvent que ces programmes servent surtout à attirer des étudiants (dans un contexte de concurrence accrue entre les universités) et à diversifier l'offre de formation. Ils doutent ainsi, plus fréquemment que leurs confrères français et italiens, que l'intérêt du séjour se situe dans l'acquisition de savoirs disciplinaires, de compétences supplémentaires et reconvertibles sur le marché du travail. Ils soulignent plutôt son rôle dans le développement de la confiance en soi de l'étudiant.

Quoiqu'il en soit, pour les étudiants Erasmus britanniques interrogés, le séjour d'une année universitaire à l'étranger se révèle sans grande incidence sur leurs 
résultats universitaires, et sans grand lien avec leurs aspirations professionnelles :

Question: "Apprendre des langues pour... ta carrière peut être... ? "

Réponse : «Euh... Pour ma carrière pas nécessairement... Je n'ai pas fait ça en termes de... Je n'ai pas fait ça pour mon futur, parce que je ne pense pas que ce soit vraiment important en Grande-Bretagne de connaître des langues d'un point de vue professionnel. Je ne me rappelle pas vraiment pourquoi je voulais faire ça... Probablement à cause de mes voyages antérieurs en Europe, mon père est hollandais, je suis en fait à moitié hollandais. »

Jack, 24 ans, doctorant en Mathématique, université de Bristol, père : inspecteur des Impôts, mère : professeur de psychologie.

"Pourquoi ai-je décidé d'aller à l'étranger? Je ne me rappelle pas... Je pensais à ça depuis longtemps, parce que j'aime voyager mais... Je pense en réalité que quand j'ai "postulé" à l'université et que j'ai remarqué qu'on pouvait aller à l'étranger (...) j'ai commencé à penser à ça et j'ai décidé que ça pouvait être marrant d'aller à l'étranger. Et ce n'est pas compliqué, parce que c'est à peu près le même diplôme».

John, 21 ans, étudiant en Master professionnel, université de Bristol, père et mère : cadres en commerce international.

Malgré ces différences (fonction des pays et institutions d'appartenance), les étudiants Erasmus européens semblent avoir des caractéristiques sociales et biographiques communes. L'origine sociale, le «prix » du temps et certaines compétences, notamment migratoires, jouent en effet un rôle important dans la décision de mobilité en cours d'études.

\section{UNE MOBILITÉ ETROITEMENT LIÉE AUXCARACTÉRISTIQUES SOCIALES ET BIOGRAPHIQUES}

Bien que les étudiants européens participent de plus en plus au programme Erasmus depuis la fin des années 80, ils ne constituent encore qu'une infime minorité. En effet, ce programme ne concerne encore qu'environ $1 \%$ des étudiants européens. Plusieurs études commanditées par la Commission européenne prennent la mesure de l'évolution au cours de ces dernières années. Mais la plupart évacuent les questions relatives aux caractéristiques sociales du «public» bénéficiaire. Ces études soulignent les effets de l'institutionnalisation de la mobilité étudiante, son impact sur la démocratisation des séjours académiques à l'étranger. La mobilité institutionnalisée permettrait ainsi, comme le souligne du reste l'étude de Jallade, Gordon et Lebeau (1996), à des étudiants d'origine modeste, d'accéder à des séjours à l'étranger, notamment par l'attribution de bourses sur critères sociaux. Peut-on pour autant affirmer, avec les auteurs de cette étude, que la situation économique influe de manière significative sur le choix de mobilité ? En se basant sur l'estimation faite par les étudiants eux-mêmes du revenu de leurs parents, les auteurs estiment que les étudiants Erasmus ne sont pas davantage privilégiés que leurs confrères sédentaires: "en réalité, $53 \%$ des étudiants sondés estiment le revenu de leurs parents équivalent ou inférieur à la moyenne. " (Jallade et al., 1996) Cette analyse est confirmée par l'enquête de Maiworm et Teichler (1997, p. 188).

Ce critère issu de l'autoreprésentation des étudiants Erasmus est-il suffisant dans l'appréciation de leur situation socio-économique? On peut en douter, a fortiori si l'on prend en compte l'extrême diversité selon les pays et les universités des systèmes d'aide publique, et par conséquent la variété des conditions sociales de mobilité offertes aux étudiants Erasmus ; en définitive, ce public apparaît globalement comme disposant de sérieux atouts sociaux. Que ce soit à Turin ou à Aix-Marseille (université de Provence), le personnel des services des relations internationales ainsi que les enseignants et étudiants rencontrés précisent que les familles doivent faire l'avance des frais engagés pour le voyage, le logement et la vie dans le pays d'accueil, puisque le paiement des bourses est conditionné au retour de la fiche de présence avec la date de départ et le tampon de l'université d'accueil. Une partie non négligeable des sommes allouées n'est donc perçue par l'étudiant qu'à son retour. Seuls $11 \%$ des étudiants Erasmus de 
l'université de Provence, en 2004-2005, disent ne pas avoir reçu d'aide financière de leurs parents pour leur séjour à l'étranger. $61 \%$ d'entre eux en ont reçu une mensuellement et $28 \%$ occasionnellement. Pour les étudiants Erasmus italiens interrogés, les proportions sont respectivement de 4\% (aucune aide), $69 \%$ (aide mensuelle) et $27 \%$ (aide occasionnelle) De plus, à l'université de Turin, les bourses de mobilité régionale ou départementale n'existent pas. Seules les facultés peuvent attribuer une aide financière, variable d'un département à l'autre et dans tous les cas jamais supérieure à une centaine d'euros par mois.

\section{Des étudiants bien dotés socialement}

Face à ces conditions objectives, les étudiants Erasmus apparaissent plutôt socialement bien armés. En effet, les résultats de notre enquête montrent une sur-représentation significative des catégories sociales favorisées parmi les étudiants Erasmus ( $c f$. Tableau 4), et ce de façon d'autant plus nette qu'elle concerne des disciplines où les langues ne font pas partie des enseignements obligatoires. À l'université de Bristol, la surreprésentation est moins évidente, car la sélection sociale à l'entrée à l'université y est déjà largement accomplie.

Le tableau 4 montre en effet que c'est dans les deux universités italienne et française que les écarts de conditions sociales (mesurées par la PCS - profession et catégorie socioprofessionnelle - des parents) entre les étudiants Erasmus et le reste de la population « témoin » sont les plus nets. Plus largement, les origines socioculturelles des étudiants apparaissent discriminantes. La majorité des étudiants Erasmus ont au moins un des deux parents qui possède un diplôme de l'enseignement supérieur, qu'il soit de «type court» (moins de 3 ans) ou de «type long» ( 3 ans ou plus). Comme pour l'origine sociale, le niveau socioculturel des parents est plus nettement différencié entre les étudiants Erasmus et les autres, à Aix-Marseille et à Turin qu'à Bristol où donc le recrutement étudiant est globalement plus socialement sélectif (cf. Tableau 5).

Les qualités scolaires des étudiants Erasmus corroborent celles observées chez leurs parents. En France, les auteurs de l'enquête de l'OURIP (Observatoire universitaire régional de l'insertion professionnelle), soulignent que les étudiants Erasmus sont plus souvent en avance ou "à l'heure " par rapport à l'ensemble des inscrits (42\% contre $24,2 \%)^{14}$. À l'université de Provence, en 2004-2005, les étudiants Erasmus étaient plus de $85 \%$ à avoir obtenu leur bac

${ }^{14}$ L'étude de l'OURIP porte sur les 2048 étudiants qui ont effectué un séjour d'études à l'étranger en 1997-98, parmi les 46463 étudiants inscrits en $2^{\mathrm{e}}$ et $3^{\mathrm{e}}$ cycles d'études dans l'une des huit universités de la région Rhône-Alpes.

Tableau 4

Origine sociale des étudiants. Universités de Bristol, Provence et Turin en 2004-2005 (en \%)

\begin{tabular}{|l|c|c|c|c|c|c|c|c|c|}
\cline { 2 - 10 } \multicolumn{1}{c|}{} & \multicolumn{3}{c|}{ Grande-Bretagne } & \multicolumn{3}{c|}{ France } & \multicolumn{3}{c|}{ Italie } \\
\cline { 2 - 10 } \multicolumn{1}{l|}{} & Erasmus & Témoin & Ens. & Erasmus & Témoin & Ens. & Erasmus & Témoin & Ens. \\
\hline $\begin{array}{l}\text { Père } \\
\text { Profession intermédiaire } \\
\text { et sup. }\end{array}$ & 90 & 72 & 80 & 64 & 30 & 51 & 63 & 37 & 49 \\
\hline $\begin{array}{l}\text { Mère } \\
\text { Profession intermédiaire } \\
\text { et sup. }\end{array}$ & 66 & 68 & 68 & 52 & 32 & 44 & 54 & 30 & 41 \\
\hline
\end{tabular}

Le tableau se lit ainsi : $90 \%$ des étudiants Erasmus britanniques ont un père exerçant une profession intermédiaire ou supérieure contre $64 \%$ des étudiants Erasmus français et $63 \%$ des étudiants Erasmus italiens (d'après les réponses aux questionnaires passés dans les trois universités étudiées).

Source : enquête par questionnaires ( $c f$. encadré 3). 
Tableau 5

Niveau d'étude des parents. Universités de Bristol, Provence et Turin en 2004-2005 (en \%)

\begin{tabular}{|l|c|c|c|c|c|c|c|c|c|}
\cline { 2 - 11 } \multicolumn{1}{c|}{} & \multicolumn{3}{c|}{ Grande-Bretagne } & \multicolumn{3}{c|}{ France } & \multicolumn{3}{c|}{ Italie } \\
\cline { 2 - 10 } \multicolumn{1}{c|}{} & Erasmus & Témoin & Ens. & Erasmus & Témoin & Ens. & Erasmus & Témoin & Ens. \\
\hline $\begin{array}{l}\text { Père avec } \\
\text { diplôme universitaire }\end{array}$ & 83 & 60 & 70 & 58 & 27 & 46 & 40 & 16 & 27 \\
\hline $\begin{array}{l}\text { Mère avec } \\
\text { diplôme universitaire }\end{array}$ & 65 & 48 & 56 & 57 & 28 & 45 & 27 & 12 & 19 \\
\hline
\end{tabular}

Le tableau se lit ainsi : $83 \%$ des étudiants Erasmus britanniques ont un père possédant un diplôme universitaire, contre $58 \%$ des étudiants Erasmus français et $40 \%$ des étudiants Erasmus italiens (d'après les réponses aux questionnaires passés dans les trois universités étudiées). Source : enquête par questionnaires ( $c f$. encadré 3 ).

en avance ou « à l'heure », contre $61 \%$ dans l'échantillon d'étudiants « sédentaires» et $62 \%$ selon les chiffres de l'OVE (Observatoire de la vie étudiante) de l'université de Provence. Chez les étudiants Erasmus, $58 \%$ avaient obtenu une mention, contre respectivement $35 \%$, parmi l'ensemble des inscrits français à l'université de Provence cette même année.

\section{De la diversité culturelle dans les usages du programme Erasmus}

Ces éclairages statistiques peuvent être complétés par les motivations antérieures et les projets postérieurs au séjour indiqués par les étudiants. Sur ce plan, de profondes disparités entre les pays et les disciplines se dessinent. Alors que l'étudiant Erasmus français semble vouloir se distinguer dans une université massifiée, l'étudiant Erasmus anglais envisage cette expérience davantage comme un voyage initiatique, à l'instar de celui qui a donné son nom au programme, Érasme de Rotterdam. Quant à l'étudiant Erasmus italien, son objectif consiste plus souvent à échapper à une insertion professionnelle difficile. Ces idéaux-types ne sont ici qu'indicatifs. Ils traduisent la diversité culturelle des usages sociaux de ce dispositif, leurs variations selon les contextes institutionnel, politique et économique des pays d'appartenance.

Si l'on s'intéresse notamment à ce qui a incité les étudiants Erasmus à opter pour le programme d'échange, on constate que l'apprentissage d'une langue étrangère constitue la raison principale évoquée, que ce soit en préparation d'une formation, d'un emploi qui requiert un certain niveau en langues ou plus simplement pour rendre son « $\mathrm{CV}$ » plus attractif. Gagner en autonomie est plus souvent cité par les étudiants italiens, mais ne constitue, dans la majorité des cas, qu'une raison secondaire. L'idée que les études à l'étranger augmentent les chances d'entrer dans des filières sélectives est également très souvent présente. Pour un grand nombre d'étudiants français et italiens interrogés, le désir de mobilité est directement associé à la construction de projets académiques ou professionnels ambitieux ou bien arrêtés. Dans leurs discours, au-delà du désir affiché de « vivre à l'étranger pendant une période assez longue », se dessinent des stratégies distinctives et promotionnelles. L'image de grandeur et de prestige, associée aux institutions étrangères de formation supérieure, représente à ce titre un des facteurs jugés importants, comme le souligne Alex, étudiant français :

«[...] ça doit aider forcément, scolairement et sur le CV évidemment c'est marqué! "Ah, vous avez passé un an en Grande-Bretagne!" on nous dit souvent dans les entretiens. Puisque [les Anglais] sont en avance sur nous, c'est même un prestige. Ca doit forcément aider, mais ce n'est pas la raison principale de mon départ. 'fin si, 'fin... Quand on vous vante les qualités d'Erasmus, on vous dit forcément, 
allez-y et puis après sur votre $C V$, au lieu de dire que vous avez fait trois ans à Marseille, vous avez fait deux ans à Marseille puis une année en GrandeBretagne, ça la fout toujours mieux, quelles que soient les notes, ça la fout toujours mieux que de dire que vous avez passé trois ans à Marseille. Ca veut dire que vous êtes capable de bouger, d'avoir des initiatives et puis c'est vrai de toute façon! »

Alex, 22 ans, Doctorant en Paléontologie, université de Paris 5 , père et mère : agriculteurs.

\section{Un passé migratoire riche}

Le programme Erasmus incite-t-il pour autant des étudiants à effectuer un séjour qu'ils n'auraient pas réalisé s'ils n'avaient pas été inscrits dans ce dispositif? En guise d'élément de réponse, on soulignera que l'étudiant Erasmus n'est pas un voyageur novice. Une des caractéristiques de cette population est en effet son passé migratoire relativement riche. Tout se passe comme si parce que leurs parents appartenant aux milieux sociaux favorisés ont fréquemment voyagé et/ou déménagé à travers diverses échelles géographiques, les étudiants Erasmus avaient conservé une aptitude certaine à la mobilité. Si l'on prend précisément comme indicateur les séjours linguistiques effectués jusqu'au moment de l'enquête, la différence entre les étudiants Erasmus et les autres est frappante ( $c f$. Tableau 6).
Là encore, le contraste entre les expériences migratoires des étudiants Erasmus par rapport au reste de la population étudiante est plus net dans les universités italienne et française étudiées où donc la sélection sociale à l'entrée a été moins forte, l'expérience migratoire des étudiants pouvant être considérée plus généralement comme une qualité socialement distinctive.

Les différences existantes dans les usages du programme Erasmus, fonction des pays et des institutions d'enseignement supérieur d'appartenance, s'accentuent avec le temps. En effet, bien qu'il existe des invariants à l'expérience Erasmus à l'étranger, les parcours post-séjour nous rappellent qu'il existe encore en Europe une grande variabilité des modalités d'insertion sur le marché du travail des jeunes.

\section{LA MOBILITÉ INSTITUTIONNALISÉE PRÉFIGURE-T-ELLE UNE «FUITE DES CERVEAUX »?}

Notre enquête nous apprend aussi qu'une part des étudiants Erasmus, après leur séjour, grâce aux contacts qu'ils ont noués et entretenus, retournent parfois dans le pays d'accueil ou émigrent dans un autre pays européen pour y prolonger leurs études

Tableau 6

Séjours linguistiques effectués avant l'entrée à l'université des étudiants des universités de Provence, Bristol et Turin (en \%).

\begin{tabular}{|l|c|c|c|c|c|c|c|c|c|}
\hline & \multicolumn{3}{|c|}{ Grande-Bretagne } & \multicolumn{3}{c|}{ France } & \multicolumn{3}{c|}{ Italie } \\
\cline { 2 - 11 } & Erasmus & Témoin & Ens. & Erasmus & Témoin & Ens. & Erasmus & Témoin & Ens. \\
\hline Aucun séjour linguistique & 30 & 78 & 57 & 25 & 78 & 47 & 31 & 80 & 58 \\
\hline Un séjour linguistique & 27 & 14 & 20 & 22 & 22 & 22 & 22 & 19 & 20 \\
\hline Deux séjours linguistiques & 16 & 5 & 10 & 26 & 0 & 16 & 27 & 0 & 12 \\
\hline $\begin{array}{l}\text { Trois séjours linguistiques } \\
\text { et }+\end{array}$ & 27 & 3 & 13 & 26 & 0 & 16 & 21 & 1 & 10 \\
\hline Total & 100 & 100 & & 100 & 100 & & 100 & 100 & 127 \\
\hline
\end{tabular}

Source : enquête par questionnaires (cf. encadré 3). 
ou y travailler. Le tableau 3 soulignait cette migration définitive dans les projets des étudiants Erasmus (le record revenant aux jeunes italiens : plus de $30 \%$ d'entre eux souhaitant, rappelons-le, entamer leur carrière à l'étranger, contre $10 \%$ des français et $7 \%$ des anglais). Lorsque la mobilité devient émigration, les facteurs attractifs sont en général toujours plus ou moins liés aux conditions de travail et opportunités de carrières qu'offre le pays d'accueil. Ce sont ces éléments attractifs que relatent les italiens rencontrés à Bristol. Quant aux raisons qui ont motivé à l'origine leur départ de leur pays, elles peuvent se résumer par les difficultés d'insertion qu'ils rencontrent face à un marché du travail national qu'ils estiment peu concurrentiel, peu motivant et tourné vers des pratiques de clientélisme (le raccomandazioni) :

«Je me suis trouvé évidemment devant un grand changement, une structure complètement différente de celle d'ici, une structure plus neuve, plus équipée [...] et ce qui m'a impressionné aussi est de voir les divers aspects de la vie, les diverses facettes [...] J'ai déjà des contacts avec des professeurs suédois, j'espère augmenter mes compétences, conclure les études, et puis qui sait, ça dépend des opportunités, si je trouve un travail, un bon travail, même à l'étranger, j'irai travailler tout de suite, si j'ai la possibilité de faire un doctorat, peut-être que je ferai un doctorat. »

Roberto, 22 ans, université de Turin, Laurea specialistica (sciences), père : commerçant ; mère : professeur des écoles.

"Mon idée est d'aller à l'étranger pour apprendre l'anglais, comme aujourd'hui le français. [...] mon idée en fait serait d'aller aux États-Unis avec une bourse d'études. Je ne sais pas s'il existe un programme, mais il y a des bourses d'études pour les diplômés qui veulent aller à l'étranger [...] il y a des possibilités. À Paris, j'ai connu un américain, parce que je vivais avec une amie italienne, elle m'a fait connaître son copain qui est américain et qui m'a dit qu'il y a des possibilités ».

Marta, 22 ans, université de Turin, Lauréa specialistica (sciences sociales), père : manager; mère : employée de banque.
Contrastant avec l'enthousiasme d'un brain movement (circulation des cerveaux) en vogue, qui se traduit notamment par l'expansion rapide des échanges universitaires, en particulier dans les nouveaux États membres de l'Europe, les jeunes travailleurs et étudiants italiens que nous avons rencontrés en Grande-Bretagne ne vivent pas toujours la mobilité de façon positive, tout particulièrement lorsqu'elle se transforme en une émigration vécue comme contrainte, en raison des conditions d'insertion professionnelles difficiles de leur pays d'origine (Caltabiano, Gianturco, 2005).

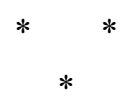

Après une vingtaine d'années d'existence, le programme Erasmus n'a semble-t-il pas atteint l'objectif officiel de réciprocité des échanges qu'il s'était fixé de façon prioritaire. En France, l'ouverture de l'enseignement supérieur à de nouveaux publics, au cours de ces dernières années, n'a fait que maintenir, voire même accentuer les différences entre les étudiants selon leur provenance sociale et scolaire (Blöss, Erlich, 2000). Si les inégalités entre les élèves se sont déplacées vers les niveaux les plus élevés de l'enseignement et par conséquent dans l'enseignement supérieur (Duru-Bellat, 2002, 2006 ; Merle, 2000), la question de la mobilité étudiante, dans un espace européen disparate, n'est pas non plus sans conséquence sur les cursus et les trajectoires des étudiants. L'expérience Erasmus est incontestablement plurielle. Pas plus que les étudiants « sédentaires », les étudiants «mobiles» ne constituent en effet un groupe homogène, mais plutôt un ensemble fractionné d'étudiants inscrits dans des circuits institutionnels différenciés.

Une des caractéristiques de la massification de l'enseignement supérieur français, réalisée au cours de ces dernières années, est d'avoir renforcé la sélection interne du système éducatif, i.e. son organisation, autour de pôles d'excellence. Dans cette organisation, les caractéristiques scolaires des étudiants se révèlent décisives. Ce sont ces qualités scolaires mais plus largement sociales qui président aux processus de sélection des étudiants Erasmus et 
orientent indirectement leur choix en matière de destination. Cette première sélection se double d'une orientation différentielle des étudiants selon les pays d'accueil. Le processus d'affinité sélective que nous évoquons désigne en effet la probabilité inégale qu'a un étudiant de choisir sa destination en fonction du niveau de prestige institutionnel de son établissement d'origine.

L'espace universitaire européen se structure ainsi dans l'ordre des inégalités de prestige entre établissements et plus largement entre aires culturelles. C'est entre autres ce que l'étude de la mobilité étudiante révèle. Le programme Erasmus est, de ce point de vue, emblématique des logiques de sélection scolaire transnationales et de leur évolution, en l'occurrence de la concurrence sociale et académique entre institutions universitaires. À qui profite, dans ces conditions, le développement des migrations internationales? Aux pays d'origine autant qu'aux pays d'accueil ? Ou bien existe-t-il un réel déséquilibre des bénéfices? Dans ce contexte, il convient de s'interroger sur les conséquences des politiques migratoires en Europe, dont le programme Erasmus constitue peu ou prou un révélateur, a fortiori quand les disparités de développement économique, social et politique au sein de l'Union européenne s'accroissent avec l'intégration d'États membres d'Europe centrale et orientale. Cette question aux multiples dimensions trouve ici quelques premiers éléments d'analyse, quand il s'agit de comprendre les logiques sociales qui ont présidé aux choix de mobilité des étudiants ainsi que le sens que prend cette expérience migratoire dans leurs trajectoires d'insertion professionnelle.

\section{Bibliographie}

Agbossou I., Carel S., Caro P. (2007), « Les échanges européens Erasmus... Accroître la mobilité des enseignants pour développer celle des étudiants », Céreq-Bref n 246, novembre.

Becher S.O., Ichino A. et Peri I. (2002), "How large is the "brain drain" from Italy?" European University Institute, dec.

Blöss T., Erlich V. (2000), « Les nouveaux acteurs de la sélection universitaire: les bacheliers technologiques en question », Revue française de sociologie, $\mathrm{n}^{\circ} 4$, octobre-décembre.

Caltabiano C., Gianturco G. (2005), Giovani oltre confine. I discendenti e gli epigoni dell'emigrazione italiana nel mondo, Roma, Carocci editore 428 p.

Cammelli A. (2001), (sous la dir. de), I laureati ERASMUS/SOCRATES anno 1999: Origine sociale, curriculum studiorum, Condizione occupazionale, Osservatorio Statistico dell'università degli Studi di Bologna, Febbraio, 59 p.

Duru-Bellat M. (2002), Les inégalités sociales à l'école : genèse et mythes, Paris, PUF, 256 p.

Duru-Bellat M. (2006), L'inflation scolaire: les désillusions de la méritocratie, Paris, Seuil, 105 p.

Fabre J., Tomasini M. (2006), «Les étudiants étrangers en France et français à l'étranger », in Données sociales - La société française.

Guarnari A. (2001), La recente emigrazione italiana in Europa: Francia, Regno Unito e Svizzera a confronto, Working paper mis en ligne en février 2001 par l'Istituto di Ricerche sulla Populazione (IRP-CNR) sous la direction de Corrado Bonifazi. 
W.P. téléchargeable sur: http:///www.irpps.cnr.it/ sito/download/wp2_01.pdf

Jallade J.-P., Gordon J., Lebeau N. (1996), Student mobility within the European Union: A statistical analysis, Rapport publié par la Commission européenne, European Institute of Education And Social Policy.

Kelo M., Teichler U., Wachter B. (2006), EURODATA - Student mobility in European Higher Education, Bonn: Lemmens.

Maiworm F., Teichler U. (1997), The Erasmus Experience: Major Findings of The ERASMUS Evaluation Research Project, European Communities Luxembourg: Office for official publication of European Communities, 209 p.

Merle P. (2000), « Le concept de démocratisation de l'institution scolaire: une typologie et sa mise à l'épreuve », in Population, "La démocratisation de l'enseignement », INED, $n^{\circ} 1$, janvier-février.

OCDE (2005), Regards sur l'éducation. Les indicateurs de l'OCDE.

OCDE (2007), Regards sur l'éducation. Les indicateurs de l'OCDE.

Pirrie A., Hamilton S., Kirk S., Davidson J. (2004), Interim Evaluation of the SOCRATES Programme in the UK, Final report, The SCRE Centre, University of Glasgow, $44 \mathrm{p}$.

Sussex Centre for Migration Research, University of Sussex, and Centre for Applied Population Research, University of Dundee (2004), Report on International student mobility, Juillet, Commissioned by HEFCE, SHEFC, HEFCW, DEL, DfES, UK Socrates Erasmus Council, HEURO, BUTEX and the British Council, Issues paper, $115 \mathrm{p}$.

\title{
Résumé
}

\section{L'autre réalité du programme Erasmus : affinité sélective entre établissements} et reproduction sociale des étudiants

\author{
Magali Ballatore et Thierry Blöss
}

Le spectre des migrations étudiantes s'est élargi au cours de ces dernières années en Europe. Son étude révèle les logiques sociales et politiques transnationales qui les mettent en œuvre. Autrement dit, les qualités individuelles que l'on prête aux étudiants migrants sont fortement liées à leurs caractéristiques scolaires et sociales, lesquelles se traduisent par une diversification croissante des parcours étudiants. Cet article a pour objet d'étude le dispositif Erasmus, programme d'échanges entre étudiants au sein de la Communauté européenne. II s'appuie sur une analyse comparative entre trois pays: la France, la Grande-Bretagne et l'Italie. II entend souligner qu'en dépit d'un principe de réciprocité des échanges, les relations concrètes entre institutions universitaires se fondent sur des logiques d'« affinités sélectives » qui à la fois révèlent le caractère relativement élitiste du dispositif et renforcent les déséquilibres migratoires déjà existants entre les pays concernés.

Mots clés :

Fuite des cerveaux, Étudiant, Mobilité internationale, Reproduction sociale, Comparaison Internationale Journal of Economic Literature: F 22, I 21, I 23 